\title{
Neuroprotection by Melatonin on Mercury Induced Toxicity in the Rat Brain
}

\author{
Mandava V. Rao, Anshita R. Purohit
}

Zoology Department, University School of Sciences, Gujarat University, Ahmedabad, India.

Email: zooldeptgu@satyam.net.in

Received May $23^{\text {rd }}, 2011$; revised July $28^{\text {th }}, 2011$; accepted September $20^{\text {th }}, 2011$.

\begin{abstract}
Free radicals are common outcome of normal aerobic cellular metabolism. In-built antioxidant system of body plays its decisive role in prevention of any loss due to free radicals. However, imbalanced defense mechanism of antioxidants and overproduction or incorporation of free radicals from environment to living systems leads to serious damage. It also attacks nervous system resulting in neural-degeneration. In order to evaluate the neurotoxic effect on the brain parts of mercury in our study, oxidative stress indices of enzymatic and non enzymatic components were measured in rats intoxicated with mercury ( $2 \mathrm{mg}$ and $4 \mathrm{mg} / \mathrm{kg}$ body weight) for 60 days to adult rats. Along with gravimetry, tissue burden was also recorded. Alterations in these indices were further supported by ultrastructural studies carried out in the brain as indicated by myelin disintegration, cell organelle alterations and neuronal loss by mercury poisoning. Treatment with the antioxidant melatonin ( $\mathrm{N}$-acetyl 5-methoxy tryptamine, $5 \mathrm{mg} / \mathrm{kg}$ ) prevented mercury exerted toxicity due to its antioxidant property. The pathological changes were also ameliorated in the brain region comparatively to support biochemical profile of brain. Thus, melatonin produced neuroprotection against mercury poisoning in rats.
\end{abstract}

Keywords: Mercuric Chloride, Melatonin, Neuroprotection, Cerebral Hemisphere, Cerebellum, Medulla Oblongata, Rats

\section{Introduction}

Inorganic mercury present in the environment is a well established toxicant to human health $[1,2]$. Mercury is released in the environment by human activity such as mining, smelting, extensive industrial and agricultural usage, combustion of fossil fuels and other industrial release. It enters the body in variety of chemical forms that are-elemental, inorganic and organic, exhibiting its toxicology characters including neurotoxicity, nephrotoxicity, reproductive toxicity and gastrointestinal toxicity with ulceration and hemorrhage [3-7]. Various mechanisms have been proposed to explain the biological toxicity of mercuric chloride, including oxidative stress. $\mathrm{Hg}^{2+}$ reacts with thiol groups $(-\mathrm{SH})$, thus depleting intracellular thiols, especially glutathione and causing oxidative stress or predisposing cells to it [8]. Mercury is a major neurotoxicant $[9,10]$; generates high levels of reactive oxygen species (ROS) and oxidative stress, depletes glutathione and thiols causing increased neurotoxicity from interactions of ROS, glutamate, and dopamine [11]; kills or inhibits production of brain tubulin cells [12-14]; inhibits production of neurotransmitters by inhibiting: cal- cium-dependent neurotransmitter release [10], dihydroteridine reductase [14], nitric oxide synthase [15], blocking neurotransmitter amino acids [16], causes abnormal migration of neurons in the cerebral cortex [17] and hence continues to pose appreciable risk to human health as evidenced by the tragic epidemics of mercury poisoning in Japan and Iraq [18]. Ingestion of mercury compounds from sea food diet is associated with aberrant central nervous system (CNS) functions [19-21]. Additionally, researchers reported that exposure to mercury can cause immune, sensory, neurological, motor and behavioral dysfunctions similar to traits associated with autism spectrum disorders (ASDs), and that these similarities extend to neuroanatomy, neurotransmitters and biochemistry. It also affects antioxidant system in the cell, resulting in loss of membrane integrity and finally cellular necrosis [22]. Other antioxidants, including ascorbic acid and vitamin $\mathrm{E}$, have been reported to be depleted in $\mathrm{HgCl}_{2}$ treated rats [23]. Basu et al. (2007) [24] demonstrated the reduction in neurochemical enzymes like cholinesterase (ChE) and monoamine oxidase (MAO) in the river otters after mercury exposure. In vivo and in 
vitro experiments were also employed by Basu et al. (2008) [25] to demonstrate inhibiting effects of mercury on the two key muscarinic cholinergic receptor subtypes (M1 and M2) in the two brain regions (occipital cortex and brain stem) of captive mink. Many experiments suggest that oxidative stress can be involved in cellular damage and that it can be implicated in the toxicity of many xenobiotics [20]. It is well known that cerebral hemisphere $(\mathrm{CH})$, cerebellum $(\mathrm{C})$ and medulla oblongata (MO) are responsible for regulating primary sensory functions and motor coordination, balance and postural stability as well as autonomous functions respectively.

Melatonin (N-acetyl-5-methoxy tryptamine) is an indoleamine secreted by the pineal gland which is located in the dorsal surface of the hypothalamus and was proven to be a free radical scavenger just over a decade ago [26]. The efficacy of melatonin in functioning to overcome oxidative stress relates to its direct free radical scavenging actions [27,28], its ability to enhance the activities of number of antioxidative enzymes [29-31], its stimulatory actions on the synthesis of another important intracellular antioxidant-glutathione [32], its efficacy to reduce electron leakage from the mitochondrial electron transport chain [33] and its synergistic interactions with the other antioxidants [34]. The interactions of melatonin membrane-bound receptors are believed to mediate endocrine and circadian rhythm effects of it. Through in vivo and in vitro studies, it was known to exhibit a potent free radical scavenging activity and also protects peroxidative damage [35]. Effects of mercury on neurotoxicity and its amelioration by melatonin are demonstrated earlier [36]. Hypothetically, melatonin plays a key role in interaction with known mercury free radical generation [37,38]. The present study was hence undertaken to evaluate the effects of mercury in relation to oxidative stress, its histological and ultrastuctural changes in relation to functions of $\mathrm{CH}, \mathrm{C}$ and MO. Further, role of melatonin on mercury exerted toxicity of brain region was also evaluated; as such study was less attempted.

\section{Materials and Methods}

\subsection{Chemicals}

Melatonin and mercuric chloride were purchased from HiMedia (Mumbai). All the chemicals used in the experiment were of the highest purity available.

\subsection{Administration of the Dose}

Adult Wistar rats (Rattus norvegicus) weighing 250 - 300 gm were procured from Cadila Pharma under the Animal Maintainance and Registeration No. 167/1999/CPCSEA from the Ministry of Social Justice and Empowerment, Govt. of India which was purely surplus outbreed stock.
The rationale of selection of male rats only is to avoid confounder such as different metabolic rate of genders (male and female), including sex hormone, lactation and pregnancy, which are inherent to females. The animals were housed under standard temperature $\left(24^{\circ} \mathrm{C} \pm 1^{\circ} \mathrm{C}\right)$, operating on a $12 \mathrm{~h}$ dark/light condition. They were fed on standard rodent chow and water ad libitum.

The experimental animals were divided into 5 groups $(\mathrm{n}=10)$. The $\mathrm{LD}_{50}$ of $\mathrm{HgCl}_{2}$ received per rat was 37 $\mathrm{mg} / \mathrm{kg}$ body weight [39]. Group I served as control and were provided with distilled water. Low dose of mercuric chloride ( $2 \mathrm{mg} / \mathrm{kg}$ body weight) was given to group II by the help of canula in the gastric gavage. High dose of mercuric chloride ( $4 \mathrm{mg} / \mathrm{kg}$ body weight) was received by Group III. Group IV was administered with melatonin ( $5 \mathrm{mg} / \mathrm{kg}$ body weight) intraperitoneally while group $\mathrm{V}$ received melatonin half an hour before the administration of high dose of $\mathrm{HgCl}_{2}$. The reason for melatonin administration 25 - 30 min before $\mathrm{HgCl}_{2}$ introduction was the rapidity of melatonin metabolism [40]. The doses were repeated daily for 60 regular days.

\subsection{Gravimetric Studies}

Body and organ weights of the rats treated with mercury were measured after and before necropsy respectively.

\subsection{Tissue Collection}

The animals were weighed and necropsy was performed after autopsy. Brain was dissected, weighed and all the 3 parts of brains, viz. cerebral hemisphere $(\mathrm{CH})$, cerebellum (C) and medulla oblongata (MO) were carefully separated, washed with ice-cold normal saline solution and placed on ice. The samples were weighed and sonicated in the respective buffers as per the protocol and the assays were performed within $24 \mathrm{~h}$ of the animal dissection and sample preparation.

\subsection{Biochemical Parameters}

The thiobarbituric acid reactive species (TBARS) levels in cerebral hemisphere, cerebellum and medulla oblongata of control and all treated animals were determined by the method of Ohkawa et al. (1979) [41]. The estimation of glutathione (GSH) and glutathione peroxidase (GPx) in all groups of rats was carried out by the method of Ellman (1959) [42] and Rotruck et al. (1973) [43] respectively. Activity of glutathione reductase was estimated by the method of Carlberg and Mannervik (1985) [44]. Glutathione-S-transferase activity was measured in the brain of control and treated group animals by the modified method of Habig et al. (1974) [45] whereas, protein carbonyl activity was done by the method of Levine et al. (1994) [46]. Levels of hydrogen peroxide were determined by the modified technique of Pick and Kei- 
sari (1981) [47]. The method of Lowry et al., (1951) [48] was employed for total protein estimation. Analysis of total ascorbic acid was done by the method of Roe and Kiether (1943) [49]. Mercury levels in the brain were estimated on mercury analyzer (ECIL, Hyderabad) using acid digestion method followed by cool vaporization of the tissue sample.

\subsection{Ultrastuctural Studies}

$\mathrm{CH}, \mathrm{C}$ and $\mathrm{MO}$ were fixed, dehydrated and cleared before embedding it for sectioning. Primary fixation was done using 3\% gluteraldehyde in 0.2 M Phosphate Buffer (pH 7.4). Secondary fixation was carried out using $1 \%$ Osmium tetroxide in the same buffer. Dehydration was carried out using ascending alcohol grade i.e., 50, 70, 80, 90 and $100 \%$ alcohol after which clearing was done with propylene oxide. The tissue was then embedded in Epoxy resin. $60 \mathrm{~nm}$ thin sections were taken on copper grid for ultrastructural studies. The grid was stained with uranyl acetate and lead citrate and observed under transmission electron microscope (TEM).

\subsection{Statistical Analysis}

Results were expressed as Mean \pm SEM. SPSS software (version 16) was used for all statistical analysis. Individual groups mean were compared by student's t-test. The data was analysed using one way analysis of variance (ANOVA) followed by Tukey's honestly significant difference (HSD) post hoc for comparing among different groups and significance was accepted when $p<0.05$. The percent amelioration was also calculated by using the following formula [50].

$\%$ amelioration $=($ pro oxidant group - respective antioxidant group)/(pro oxidant group - Control $) \times 100$.

\section{Results}

In the present study, the chronic administration of $\mathrm{HgCl}_{2}$ to rats caused an imbalance in oxidative stress as monitored by means of antioxidant indices, which is confronted by transmission electron microscopic studies on the brain.

\subsection{Effect of MC on Gravimetric Data and Mercury Levels}

\subsubsection{Body Weights}

Treatment of MC to rats significantly $(p<0.05)$ reduced the body weight. Besides weight loss, the animal showed signs of toxication like yellowish fur and a cachectic appearance. High dose of MC resulted in further significant reduction $(p<0.001)$ within the dose period of 60 days. The amelioration obtained in the body weight after melatonin supplementation was 50\% (Table 1).

\subsubsection{Organ Weights}

Total weight of brain, cerebral hemisphere $(\mathrm{CH})$, cerebellum (C) and medulla oblongata (MO) were weighed after sacrifice to nearest milligram by digital balance. Mercury treatment reduced the organ weights of rats in a dose dependent way, where high dose brought about a statistically significant $(p<0.001)$ reduction in total weight, $\mathrm{CH}, \mathrm{C}$ and $\mathrm{MO}$. Melatonin co-administration gave $45.5 \%, 36.9 \%, 31.2 \%$ and $70 \%$ amelioration respectively (Table 1).

\subsubsection{Mercury Levels}

Mercury retention was measured with the help of mercury analyzer where a significant retention $(p<0.001)$ in the mercury levels were noted in $\mathrm{C}$ and MO in treated rats and $\mathrm{CH}$ had also a significant retention $(p<0.05)$ at high dose only. Amelioration was $87.2 \%, 84.8 \%$ and 90.6\% in $\mathrm{CH}, \mathrm{C}$ and $\mathrm{MO}$ respectively (Table 2).

\subsection{Effect of MC on Enzymatic and Non Enzymatic Antioxidants}

\subsubsection{MDA, Catalase, Superoxide Dismutase and Ascorbic Acid Levels}

A significant $(p<0.001 ; p<0.05)$ increase in levels of MDA and catalase activity were observed in the MC treated groups in $\mathrm{CH}, \mathrm{C}$ and $\mathrm{MO}$, as compared to the control group. However, the amelioration obtained after melatonin administration for MDA was $68.4 \%, 50.8 \%$ and $58.7 \%$ respectively whereas, for catalase was $62.3 \%$, $20 \%$ and $26.9 \%$ respectively. Treatment with MC brought about a decline in SOD $(p<0.001)$ and TAA levels of $\mathrm{CH}$, $\mathrm{C}$ and $\mathrm{MO}$ by mercury feeding. The amelioration was $41 \%, 20.7 \%$ and $25.8 \%$ respectively for SOD and $15.2 \%$, $14.1 \%$ and $22.3 \%$ respectively for total ascorbic acid after MLT co-administration (Table 3).

\subsubsection{GSH, GPx and GR}

Treatment with MC induced a significant $(p<0.001)$ fall in GSH levels in the brain compared with control giving $47.1 \%$. 68.8\% and $83.8 \%$ amelioration after melatonin supplements to the rats. Similarly, Glutathione peroxidase (GPx) activity declined in all brain regions. A significant $(p<0.001)$ reduction in its values were noted in cerebral hemisphere, cerebellum and medulla oblongata in mercury treated rats. A significant $(p<0.05)$ reduction of GPx activity in C and MO were recorded at combination level. The activity of GR recorded a highly significant $(p<0.001)$ decline in $\mathrm{CH}, \mathrm{C}$ and $\mathrm{MO}$ by $\mathrm{MC}$ treatment for 60 days. With melatonin and mercury combination, a reduction $(p<0.01)$ was noticed in $\mathrm{CH}$ and $\mathrm{C}$ only (Table 4).

3.2.3. GST, Protein Carbonyl, $\mathrm{H}_{2} \mathrm{O}_{2}$ and Total (-SH) Glutathione-S-transferase (GST) showed increase $(p<$ 
Table 1. Body and organ weights of control and experimental groups.

\begin{tabular}{|c|c|c|c|c|c|c|c|}
\hline \multirow{2}{*}{ Parameters } & \multirow{2}{*}{ Control } & \multicolumn{2}{|c|}{$\mathrm{HgCl}_{2}$} & \multirow{2}{*}{ Melatonin } & \multirow{2}{*}{ Melatonin $+\mathrm{HD}$} & \multirow{2}{*}{ Anova } & \multirow{2}{*}{ Amelioration (\%) } \\
\hline & & LD & $\mathrm{HD}$ & & & & \\
\hline Total body weights (gm) & $425 \pm 11.10$ & $354 \pm 4.00^{\mathrm{NS}}$ & $279 \pm 6.40^{*}$ & $347 \pm 9.94^{\mathrm{NS}}$ & $306 \pm 4.00^{\mathrm{NS}}$ & $51.86^{\#}$ & 50.00 \\
\hline Total brain weights (mg) & $2163.4 \pm 14.6$ & $2044.1 \pm 10.7^{+}$ & $1992 \pm 3.93^{+}$ & $2182 \pm 6.65^{\mathrm{NS}}$ & $2070 \pm 13.7^{* *}$ & $56.28^{\#}$ & 45.50 \\
\hline Cerebral hemisphere (mg) & $1332 \pm 15.39$ & $1258 \pm 17.4^{*}$ & $1148 \pm 17.9^{+}$ & $1349 \pm 17.48^{\mathrm{NS}}$ & $1216 \pm 15.32^{\mathrm{NS}}$ & $24.60^{\#}$ & 36.90 \\
\hline Cerebellum (mg) & $480.0 \pm 4.10$ & $440.7 \pm 9.92^{* *}$ & $425.0 \pm 3.544^{+}$ & $475.2 \pm 15.2^{\mathrm{NS}}$ & $442.2 \pm 8.26^{\mathrm{NS}}$ & $16.54^{\#}$ & 31.27 \\
\hline Medulla oblongata (mg) & $441.0 \pm 8.47$ & $434.0 \pm 10.00^{* *}$ & $421.1 \pm 9.40^{+}$ & $460.0 \pm 11.70^{\mathrm{NS}}$ & $435.0 \pm 11.30^{\mathrm{NS}}$ & $7.133^{\#}$ & 70.00 \\
\hline
\end{tabular}

Table 2. Mercury levels measured in control and experimental groups.

\begin{tabular}{|c|c|c|c|c|c|c|c|c|}
\hline \multirow{2}{*}{ Parameters } & \multirow{2}{*}{ Organs } & \multirow{2}{*}{ Control } & \multicolumn{2}{|c|}{$\mathrm{HgCl}_{2}$} & \multirow{2}{*}{ Melatonin } & \multirow{2}{*}{ Melatonin + HD } & \multirow{2}{*}{ Anova } & \multirow{2}{*}{ Amelioration (\%) } \\
\hline & & & LD & HD & & & & \\
\hline \multirow{3}{*}{$\begin{array}{l}\text { Mercury Levels } \\
\text { ( } \mu \mathrm{g} / \mathrm{gm} \text { tissue } \\
\text { weight) }\end{array}$} & $\mathrm{CH}$ & $0.30 \pm 0.10$ & $5.59 \pm 0.05^{+}$ & $9.67 \pm 0.14^{+}$ & $0.09 \pm 0.14^{\mathrm{NS}}$ & $2.72 \pm 0.15^{\mathrm{NS}}$ & $2.78^{\#}$ & 87.20 \\
\hline & $\mathrm{C}$ & $0.27 \pm 0.09$ & $4.89 \pm 0.04^{+}$ & $8.73 \pm 0.06^{+}$ & $0.02 \pm 0.20^{\mathrm{NS}}$ & $2.57 \pm 0.08^{\mathrm{NS}}$ & $3.57^{\#}$ & 84.84 \\
\hline & MO & $0.24 \pm 0.12$ & $4.09 \pm 0.09^{+}$ & $7.74 \pm 0.08^{+}$ & $0.00 \pm 0.07^{\mathrm{NS}}$ & $2.03 \pm 0.17^{\mathrm{NS}}$ & $3.75^{\#}$ & 90.63 \\
\hline
\end{tabular}

Values are Mean \pm S.E., $\mathrm{HgCl}_{2}=$ Mercuric Chloride; $\mathrm{LD}=$ Low Dose; $\mathrm{HD}=$ High Dose; $\mathrm{CH}=$ Cerebral Hemisphere; $\mathrm{C}=$ Cerebellum; $\mathrm{MO}=\mathrm{Medulla}$ Oblon gata; NS = Non Significant; ${ }^{*} p<0.05 ;{ }^{* *} p<0.01 ;{ }^{+} p<0.001$; significant analysis of variance at ${ }^{\#} p \leq 0.05$.

Table 3. Enzymes of antioxidant system in brain of control and treated animals.

\begin{tabular}{|c|c|c|c|c|c|c|c|c|}
\hline \multirow{2}{*}{ Parameters } & \multirow{2}{*}{ Organs } & \multirow{2}{*}{ Control } & \multicolumn{2}{|c|}{$\mathrm{HgCl}_{2}$} & \multirow{2}{*}{ Melatonin } & \multirow{2}{*}{ Melatonin $+\mathrm{HD}$} & \multirow{2}{*}{ Anova } & \multirow{2}{*}{ Amelioration (\%) } \\
\hline & & & LD & HD & & & & \\
\hline \multirow{3}{*}{$\begin{array}{c}\text { LPO } \\
\text { (nanomoles of } \\
\text { MDA/100 mg } \\
\text { tissue weight) }\end{array}$} & $\mathrm{CH}$ & $37.22 \pm 3.54$ & $58.11 \pm 1.46^{+}$ & $66.83 \pm 5.60^{+}$ & $45.55 \pm 2.28^{\mathrm{NS}}$ & $46.55 \pm 2.63^{*}$ & $11.39^{\#}$ & 68.49 \\
\hline & $\mathrm{C}$ & $40.76 \pm 2.28$ & $57.32 \pm 2.00^{+}$ & $71.77 \pm 1.62^{+}$ & $39.18 \pm 8.20^{\mathrm{NS}}$ & $56.00 \pm 6.41^{\mathrm{NS}}$ & $7.49^{\#}$ & 50.85 \\
\hline & MO & $37.5 \pm 1.77$ & $42.94 \pm 0.63^{* *}$ & $51.05 \pm 1.66^{+}$ & $38.87 \pm 1.62^{\mathrm{NS}}$ & $43.09 \pm 1.04^{* *}$ & $16.48^{\#}$ & 58.74 \\
\hline \multirow{3}{*}{$\begin{array}{c}\text { SOD } \\
\text { (units/mg protein) }\end{array}$} & $\mathrm{CH}$ & $2.277 \pm 0.07$ & $1.499 \pm 0.04^{+}$ & $1.277 \pm 0.08^{+}$ & $1.99 \pm 0.04^{\mathrm{NS}}$ & $1.68 \pm 0.07^{* *}$ & $2.58^{\#}$ & 41.00 \\
\hline & $\mathrm{C}$ & $1.733 \pm 0.04$ & $0.975 \pm 0.01^{+}$ & $0.873 \pm 0.03^{+}$ & $1.271 \pm 0.05^{\mathrm{NS}}$ & $1.051 \pm 0.03^{*}$ & $4.19^{\#}$ & 20.77 \\
\hline & MO & $2.306 \pm 0.09$ & $1.145 \pm 0.02^{+}$ & $1.069 \pm 0.04^{+}$ & $2.054 \pm 0.01^{\mathrm{NS}}$ & $1.38 \pm 0.15^{\mathrm{NS}}$ & $1.77^{\#}$ & 25.80 \\
\hline \multirow{3}{*}{$\begin{array}{c}\text { Catalase } \\
\left(\mu \text { moles } \mathrm{H}_{2} \mathrm{O}_{2}\right. \\
\text { consumed } / \\
\mathrm{min} / \text { mg protein })\end{array}$} & $\mathrm{CH}$ & $18.37 \pm 1.27$ & $17.67 \pm 3.7^{\mathrm{NS}}$ & $14.25 \pm 3.33^{*}$ & $21.81 \pm 4.41^{\mathrm{NS}}$ & $16.82 \pm 2.34^{\mathrm{NS}}$ & $0.42^{\#}$ & 62.37 \\
\hline & $\mathrm{C}$ & $30.5 \pm 3.47$ & $26.69 \pm 0.9^{\mathrm{NS}}$ & $24.17 \pm 2.13^{*}$ & $30.72 \pm 4.6^{\mathrm{NS}}$ & $25.44 \pm 2.99^{\mathrm{NS}}$ & $0.67^{\#}$ & 20.06 \\
\hline & MO & $34.94 \pm 3.69$ & $31.32 \pm 2.73^{\mathrm{NS}}$ & $25.16 \pm 4.83^{*}$ & $35.2 \pm 3.78^{\mathrm{NS}}$ & $27.8 \pm 4.2^{\mathrm{NS}}$ & $0.78^{\#}$ & 26.99 \\
\hline \multirow{3}{*}{$\begin{array}{c}\text { TAA } \\
\text { (mg/gm tissue } \\
\text { weight) }\end{array}$} & $\mathrm{CH}$ & $2.46 \pm 0.65$ & $2.22 \pm 0.42^{\mathrm{NS}}$ & $1.72 \pm 0.07^{\mathrm{NS}}$ & $2.75 \pm 0.44^{\mathrm{NS}}$ & $1.83 \pm 0.21^{\mathrm{NS}}$ & $0.91^{\#}$ & 15.20 \\
\hline & $\mathrm{C}$ & $2.85 \pm 0.68$ & $2.60 \pm 0.59^{\mathrm{NS}}$ & $1.51 \pm 0.11^{\mathrm{NS}}$ & $2.94 \pm 0.14^{\mathrm{NS}}$ & $1.70 \pm 0.42^{\mathrm{NS}}$ & $2.14^{\#}$ & 14.17 \\
\hline & MO & $2.62 \pm 0.31$ & $2.14 \pm 0.28^{\mathrm{NS}}$ & $1.41 \pm 0.12^{\mathrm{NS}}$ & $2.66 \pm 0.13^{\mathrm{NS}}$ & $1.68 \pm 0.28^{\mathrm{NS}}$ & $4.47^{\#}$ & 22.31 \\
\hline
\end{tabular}

Values are Mean \pm S.E., $\mathrm{HgCl}_{2}=$ Mercuric Chloride; $\mathrm{LD}=$ Low Dose; $\mathrm{HD}=$ High Dose; $\mathrm{CH}=$ Cerebral Hemisphere; $\mathrm{C}=\mathrm{Cerebellum}$ and $\mathrm{MO}=\mathrm{Medulla}$ Oblongata; LPO = Lipid Peroxidation; SOD = Superoxide Dismutase; NS = Non Significant; ${ }^{*} p<0.05 ;{ }^{* *} p<0.01 ;{ }^{+} p<0.001$; significant analysis of variance at ${ }^{\#} p \leq 0.05$. 
Table 4. Non enzymatic and enzymatic antioxidant system in brain of experimental animals.

\begin{tabular}{|c|c|c|c|c|c|c|c|c|}
\hline \multirow{2}{*}{ Parameters } & \multirow{2}{*}{ Organs } & \multirow{2}{*}{ Control } & \multicolumn{2}{|c|}{$\mathrm{HgCl}_{2}$} & \multirow{2}{*}{ Melatonin } & \multirow{2}{*}{ Melatonin + HD } & \multirow{2}{*}{ Anova } & \multirow{2}{*}{$\begin{array}{c}\text { Amelioration } \\
(\%)\end{array}$} \\
\hline & & & LD & HD & & & & \\
\hline \multirow{3}{*}{$\begin{array}{c}\mathrm{GSH} \\
(\mu \mathrm{g} / 100 \mathrm{mg} \\
\text { tissue weight })\end{array}$} & $\mathrm{CH}$ & $5.40 \pm 0.11$ & $3.62 \pm 0.02^{+}$ & $2.28 \pm 0.08^{+}$ & $5.54 \pm 0.11^{\mathrm{NS}}$ & $3.75 \pm 0.05^{* *}$ & $10.7^{\#}$ & 47.11 \\
\hline & $\mathrm{C}$ & $5.54 \pm 0.06$ & $4.1 \pm 0.04^{+}$ & $2.52 \pm 0.08^{+}$ & $5.61 \pm 0.08^{\mathrm{NS}}$ & $4.60 \pm 0.01^{* *}$ & $16.81^{\#}$ & 68.87 \\
\hline & MO & $4.22 \pm 0.05$ & $3.38 \pm 0.07^{+}$ & $1.38 \pm 0.12^{+}$ & $4.50 \pm 0.03^{\mathrm{NS}}$ & $3.76 \pm 0.06^{* *}$ & $13.89^{\#}$ & 83.80 \\
\hline \multirow{3}{*}{$\begin{array}{c}\text { GPx } \\
\text { (GSH consumed/ } \\
\mathrm{min} / \mathrm{mg} \text { protein) }\end{array}$} & $\mathrm{CH}$ & $0.071 \pm 0.015$ & $0.011 \pm 0.006^{+}$ & $0.006 \pm 0.001^{+}$ & $0.047 \pm 0.002^{\mathrm{NS}}$ & $0.016 \pm 0.001^{\mathrm{NS}}$ & $2.04^{\#}$ & 15.38 \\
\hline & $\mathrm{C}$ & $0.063 \pm 0.014$ & $0.028 \pm 0.002^{*}$ & $0.015 \pm 0.003^{* *}$ & $0.050 \pm 0.002^{\mathrm{NS}}$ & $0.022 \pm 0.002^{*}$ & $2.75^{\#}$ & 14.93 \\
\hline & MO & $0.087 \pm 0.004$ & $0.053 \pm 0.001^{+}$ & $0.027 \pm 0.005^{+}$ & $0.082 \pm 0.001^{\mathrm{NS}}$ & $0.042 \pm 0.005^{*}$ & $9.51^{\#}$ & 25.00 \\
\hline \multirow{3}{*}{$\begin{array}{c}\text { GR } \\
\text { (moles NADPH } \\
\text { oxidized } / \mathrm{min} / \mathrm{mg} \\
\text { protein) }\end{array}$} & $\mathrm{CH}$ & $54.95 \pm 2.60$ & $28.05 \pm 0.24^{+}$ & $25.49 \pm 0.30^{+}$ & $55.98 \pm 2.90^{\mathrm{NS}}$ & $28.89 \pm 1.27^{* *}$ & $3.31^{\#}$ & 11.54 \\
\hline & $\mathrm{C}$ & $31.72 \pm 0.33$ & $19.30 \pm 0.40^{+}$ & $14.27 \pm 0.41^{+}$ & $30.28 \pm 0.32^{\mathrm{NS}}$ & $19.00 \pm 0.39^{*}$ & $19.3^{\#}$ & 27.10 \\
\hline & MO & $51.65 \pm 2.42$ & $32.80 \pm 2.05^{+}$ & $25.54 \pm 0.05^{+}$ & $51.73 \pm 0.69^{\mathrm{NS}}$ & $30.75 \pm 0.69^{\mathrm{NS}}$ & $0.07^{\#}$ & 19.98 \\
\hline
\end{tabular}

Values are Mean \pm S.E., $\mathrm{HgCl}_{2}=$ Mercuric Chloride; $\mathrm{LD}=$ Low Dose; $\mathrm{HD}=$ High Dose $\mathrm{CH}=\mathrm{Cerebral}$ Hemisphere; $\mathrm{C}=\mathrm{Cerebellum}$ and $\mathrm{MO}=\mathrm{Medulla}$ Oblongata; GSH = Glutathione-SH; GPx = Glutathione Peroxidase; GR = Glutathione Reductase; NS = Non Significant; ${ }^{*} p<0.05 ;{ }^{* *} p<0.01 ;{ }^{+} p<0.001$; significant analysis of variance at ${ }^{\#} p \leq 0.05$.

$0.001 ; p<0.05)$ in pro-oxidant treated groups. Antioxidant supplement to the intoxicated rat proved efficient in ameliorating $\mathrm{HgCl}_{2}$ induced toxicity comparable to control values giving $54.2 \%, 64.1 \%$ and $40 \%$ amelioration. Protein carbonyl levels were significantly $(p<0.005)$ increased after the treatment. The percent amelioration after melatonin administration was $81.8 \%, 43.7 \%$ and $33.7 \%$ respectively. Hydrogen peroxide $\left(\mathrm{H}_{2} \mathrm{O}_{2}\right)$ levels were found to be increased by MC administration. MLT supplementation gave $67.9 \%, 78.4 \%$ and $67.2 \%$ amelioration in $\mathrm{CH}, \mathrm{C}$ and $\mathrm{MO}$ respectively. Total sulfhydryl groups showed a significant decline $(p<0.01)$ in $\mathrm{CH}, \mathrm{C}$ and $\mathrm{MO}$ with mercury intoxication which, after the administration of melatonin gave $65.2 \%, 20.4 \%$ and $43.9 \%$ amelioration respectively (Table 5).

\subsection{Effect of MC on Ultrastructure of Brain}

The Transmission electron (TE) micrographs of rat brain revealed that myelinated nerve (My) of control rat brain showed internal (i) and external (e) mesa axons with mitochondria (Mi) and vesicles inside it. Mitochondria outside the axon appear to be normal including occurrence of occasional ribosomes (Figure 1). On the other hand, electron micrograph of a treated rat brain indicated distorted axon with discontinuous myelin sheath $(*)$. A reduction in number of vesicles (Ve) and mitochondria in the axon were noticed. The mitochondria (Mi) were also swollen in the matrix (Figure 2). In the micrograph of rat brain co-treated with melatonin, the mitochondria appeared normal with dense vesicular components which appeared to that of control rats (Figure 3). The nucleus (N) of the control rat brain cell has continuous nuclear membrane (NM) with diffused chromatin material inside it (Figure 4). But the nucleus turned blebbed in the treated rats and the nuclear membrane becomes discontinuous and puffed (\#) at irregular intervals with increased intracellular space (S) (Figure 5). These changes of the nucleus were not observed in the brain of mercury treated rats co-administered with melatonin but the intracellular spaces are still persistent (Figure 6).

\section{Discussion}

Environmental and occupational pollution leads to accumulation of heavy metals like mercury resulting into serious health problems [51-53]. Organic and inorganic mercury salts have been proved to be potent toxic agents in animals including human. Chronic neurotoxicity is reported at concentration $>35 \mu \mathrm{g} / \mathrm{L}$, whereas acute toxicity occurs at concentrations $>200 \mu \mathrm{g} / \mathrm{L}$ [14]. $\mathrm{HgCl}_{2}$ ingestion has been shown to reduce body and organ weights in laboratory animals [54]. Similarly, in the present study, a significant reduction in body and brain weights was observed. Besides weight loss, the animal showed signs of toxication like yellowish fur and a cachectic appearance. Numerous studies indicate that mercuric ions interact with glutathione (GSH) and other $(-\mathrm{SH})$ groups in the presence of hydrogen peroxide, leading to the generation of reactive oxygen species (ROS). These ROS subsequently include lipid peroxidation measured by thiobarbituric acid reaction for malondialdehyde in the 
Table 5. Parameters studied in brain for Oxidative stress and enzymatic antioxidant system.

\begin{tabular}{|c|c|c|c|c|c|c|c|c|}
\hline \multirow{2}{*}{ Parameters } & \multirow{2}{*}{ Organs } & \multirow{2}{*}{ Control } & \multicolumn{2}{|c|}{$\mathrm{HgCl}_{2}$} & \multirow{2}{*}{ Melatonin } & \multirow{2}{*}{ Melatonin + HD } & \multirow{2}{*}{ Anova } & \multirow{2}{*}{$\begin{array}{l}\text { Amelioration } \\
(\%)\end{array}$} \\
\hline & & & LD & HD & & & & \\
\hline \multirow{3}{*}{$\begin{array}{l}\text { GST (units/mg } \\
\text { protein) }\end{array}$} & $\mathrm{CH}$ & $0.0058 \pm 0.0003$ & $0.00594 \pm 0.0002^{\mathrm{NS}}$ & $0.00658 \pm 0.0002^{*}$ & $0.00592 \pm 0.0002^{\mathrm{NS}}$ & $0.00612 \pm 0.0001^{\mathrm{NS}}$ & $0.82^{\#}$ & 54.28 \\
\hline & $\mathrm{C}$ & $0.0035 \pm 0.0001$ & $0.0044 \pm 0.0001^{+}$ & $0.00545 \pm 0.0002^{+}$ & $0.00352 \pm 0.0002^{\mathrm{NS}}$ & $0.0120 \pm 0.0012^{\mathrm{NS}}$ & $1.77^{\#}$ & 64.10 \\
\hline & MO & $0.00256 \pm 0.001$ & $0.0027 \pm 0.0003^{\mathrm{NS}}$ & $0.00296 \pm 0.0001^{*}$ & $0.00260 \pm 0.0001^{\mathrm{NS}}$ & $0.00280 \pm 0.0002^{\mathrm{NS}}$ & $4.19^{\#}$ & 40.00 \\
\hline \multirow{3}{*}{$\begin{array}{c}\text { Protein } \\
\text { Carbonyl (nmol/ } \\
\text { mg protein) }\end{array}$} & $\mathrm{CH}$ & $10.7 \pm 0.80$ & $11.7 \pm 0.95^{\mathrm{NS}}$ & $12.9 \pm 0.97^{*}$ & $09.7 \pm 0.50^{\mathrm{NS}}$ & $11.1 \pm 0.40^{\mathrm{NS}}$ & $1.85^{\#}$ & 81.80 \\
\hline & $\mathrm{C}$ & $09.3 \pm 0.70$ & $10.0 \pm 0.29^{\mathrm{NS}}$ & $10.9 \pm 0.07^{*}$ & $09.4 \pm 0.60^{\mathrm{NS}}$ & $10.2 \pm 0.13^{\mathrm{NS}}$ & $6.02^{\#}$ & 43.75 \\
\hline & MO & $10.2 \pm 0.33$ & $10.7 \pm 0.30^{\mathrm{NS}}$ & $11.1 \pm 0.28^{*}$ & $10.0 \pm 0.70^{\mathrm{NS}}$ & $10.8 \pm 0.30^{\mathrm{NS}}$ & $1.05^{\#}$ & 33.70 \\
\hline \multirow{3}{*}{$\begin{array}{c}\mathrm{H}_{2} \mathrm{O}_{2} \\
\left(\mu \mathrm{M} \mathrm{of} \mathrm{H}_{2} \mathrm{O}_{2}\right. \\
\text { formed/100 mg } \\
\text { tissue wt) }\end{array}$} & $\mathrm{CH}$ & $69.6 \pm 5.64$ & $77.2 \pm 5.31^{\mathrm{NS}}$ & $80.2 \pm 1.20^{\mathrm{NS}}$ & $64.0 \pm 5.02^{\mathrm{NS}}$ & $73.0 \pm 3.06^{\mathrm{NS}}$ & $1.68^{\#}$ & 67.90 \\
\hline & $\mathrm{C}$ & $55.2 \pm 4.70$ & $58.8 \pm 1.74^{\mathrm{NS}}$ & $75.6 \pm 3.42^{\mathrm{NS}}$ & $54.4 \pm 3.48^{\mathrm{NS}}$ & $59.6 \pm 2.03^{\mathrm{NS}}$ & $2.18^{\#}$ & 78.43 \\
\hline & MO & $50.6 \pm 5.29$ & $66.2 \pm 4.56^{\mathrm{NS}}$ & $74.4 \pm 3.61^{\mathrm{NS}}$ & $48.2 \pm 1.15^{\mathrm{NS}}$ & $58.4 \pm 4.16^{\mathrm{NS}}$ & $4.27^{\#}$ & 67.20 \\
\hline \multirow{3}{*}{$\begin{array}{c}\text { Total }(-\mathrm{SH}) \\
(\mu \mathrm{g} / 100 \mathrm{mg} \\
\text { fresh tissue wt) }\end{array}$} & $\mathrm{CH}$ & $2.10 \pm 0.17$ & $1.59 \pm 0.09^{*}$ & $1.47 \pm 0.05^{* *}$ & $1.54 \pm 0.06^{\mathrm{NS}}$ & $1.06 \pm 0.12^{* *}$ & $7.12^{\#}$ & 65.23 \\
\hline & $\mathrm{C}$ & $1.66 \pm 0.12$ & $1.54 \pm 0.12^{\mathrm{NS}}$ & $1.22 \pm 0.11^{*}$ & $1.68 \pm 0.1^{\mathrm{NS}}$ & $1.31 \pm 0.05^{\mathrm{NS}}$ & $2.69^{\#}$ & 20.45 \\
\hline & MO & $1.97 \pm 0.14$ & $1.65 \pm 0.04^{\mathrm{NS}}$ & $1.42 \pm 0.17^{*}$ & $2.01 \pm 0.01^{\mathrm{NS}}$ & $1.66 \pm 0.077$ & $1.75^{\#}$ & 43.90 \\
\hline
\end{tabular}

Values are Mean \pm S.E., $\mathrm{HgCl}_{2}=$ Mercuric Chloride; $\mathrm{LD}=$ Low Dose; $\mathrm{HD}=$ High Dose; $\mathrm{CH}=$ Cerebral Hemisphere; $\mathrm{C}=\mathrm{Cerebellum}$ and $\mathrm{MO}=\mathrm{Medulla}$ Oblongata; GST = Glutathione S-Transferase; $\mathrm{H}_{2} \mathrm{O}_{2}=$ Hydrogen Peroxide; Total $(-\mathrm{SH})=$ Total Sulphydryl Groups. NS = Non Significant; ${ }^{*} p<0.05 ;{ }^{* *} p<0.01$; ${ }^{+} p<0.001$; significant analysis of variance at ${ }^{\#} p \leq 0.05$.

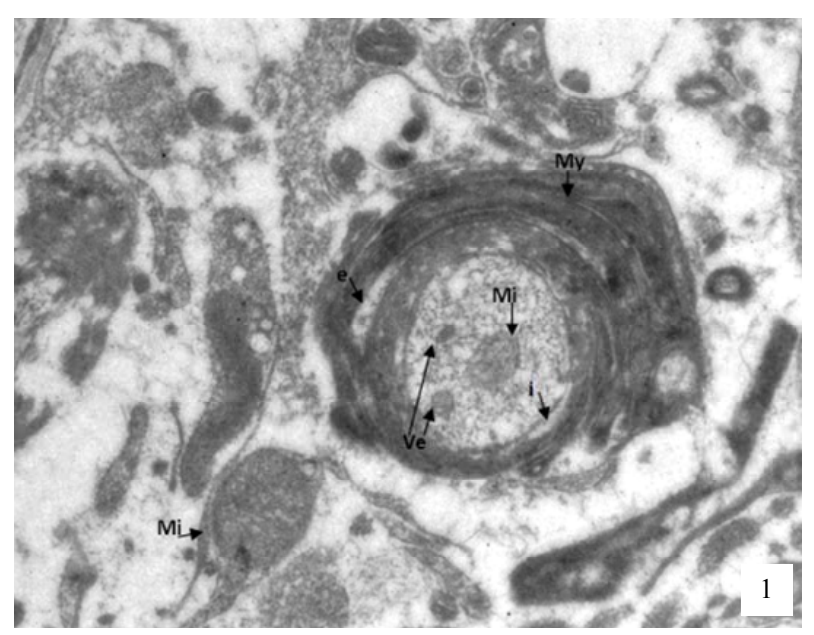

Figure 1. Electron micrograph of control rat brain showing normal myelinated nerve with external (e) and internal (i) mesa axons as well as mitochondria (Mi) and vesicles (Ve) present inside the axon $(\times 6300)$.

brain. Lipid peroxidation is known to be one of the molecular mechanisms for cell injury in acute mercury poisoning and is associated with a decrease in cellular antioxidants such as glutathione ascorbate, superoxide dismutase (SOD) and catalase (CAT) $[55,56]$. Catalase ac-

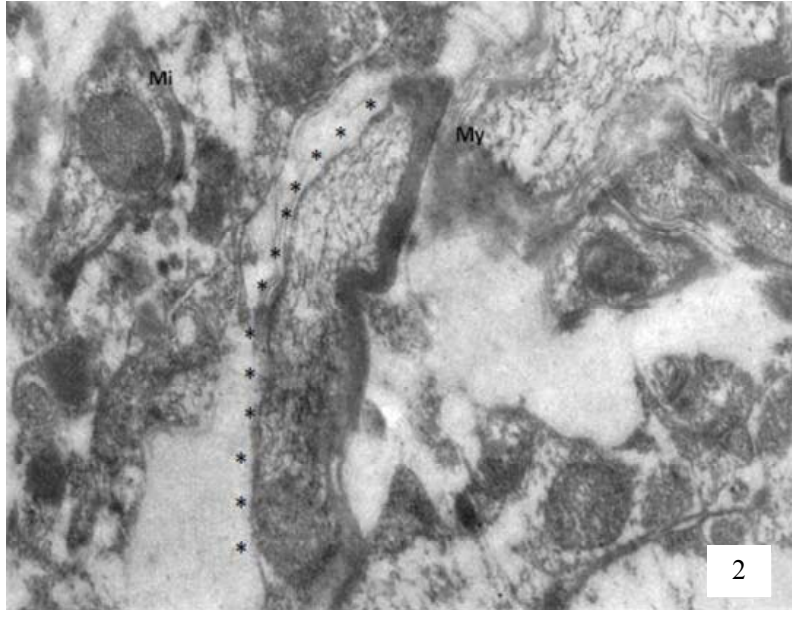

Figure 2. Electron micrograph of treated rat brain showing discontinuous myelin sheath (*) around the distorted axon (×6300).

tivity was found to be decreased in this study. This enzyme is responsible for balancing the production of $\mathrm{H}_{2} \mathrm{O}_{2}$ and superoxide radicals. As CAT activity decreases, the level of $\mathrm{H}_{2} \mathrm{O}_{2}$ is found to increase in the present data as justified by reduced levels of this enzyme. The decreased activity of SOD further indicated an increased superoxide 


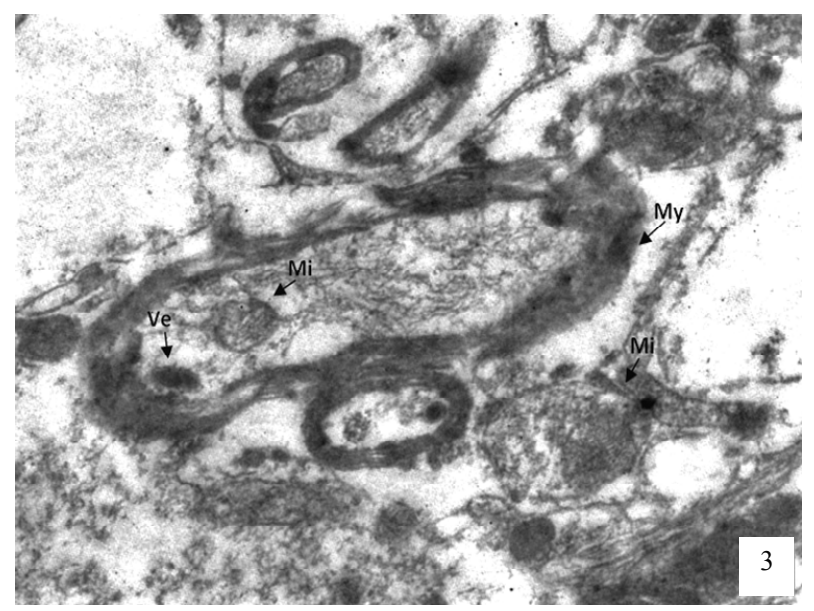

Figure 3. Electron micrograph of mercury and melatonin treated rat where myelin sheath is still less continuous (×6300).

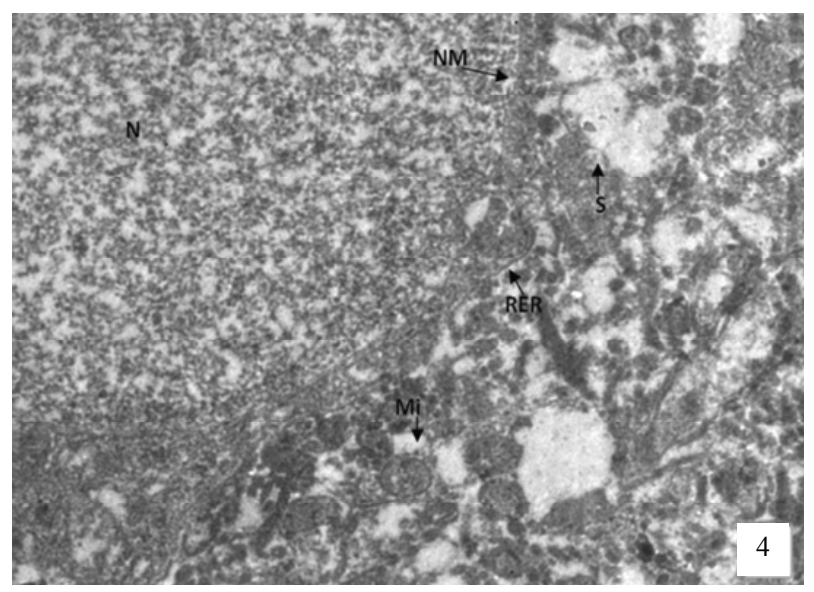

Figure 4. Electron micrograph of control rat brain nucleus (N) with nuclear membrane $(\mathrm{NM})(\times 5000)$.

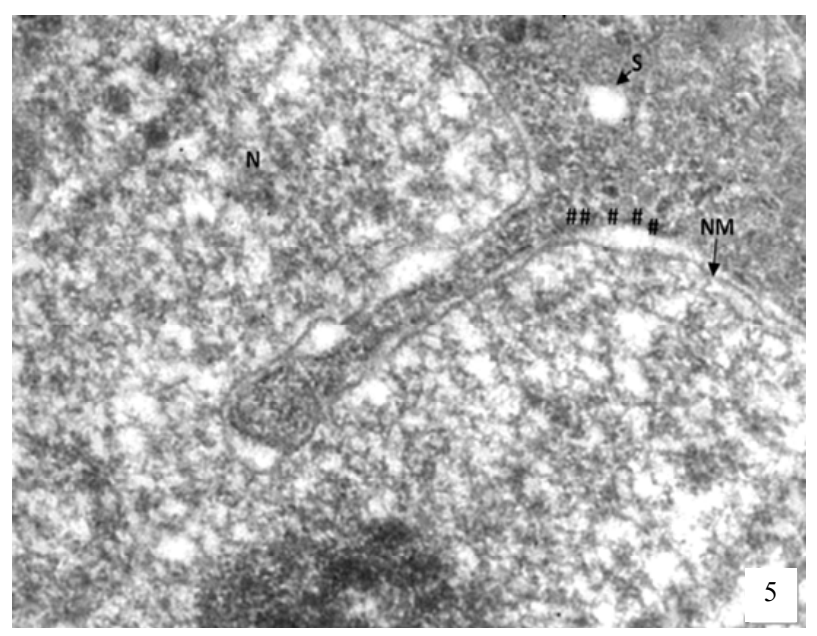

Figure 5. Electron micrograph of treated rat brain nucleus with puffed nuclear membrane (\#) at irregular intervals and large intracellular spaces $(S)(\times 5000)$.

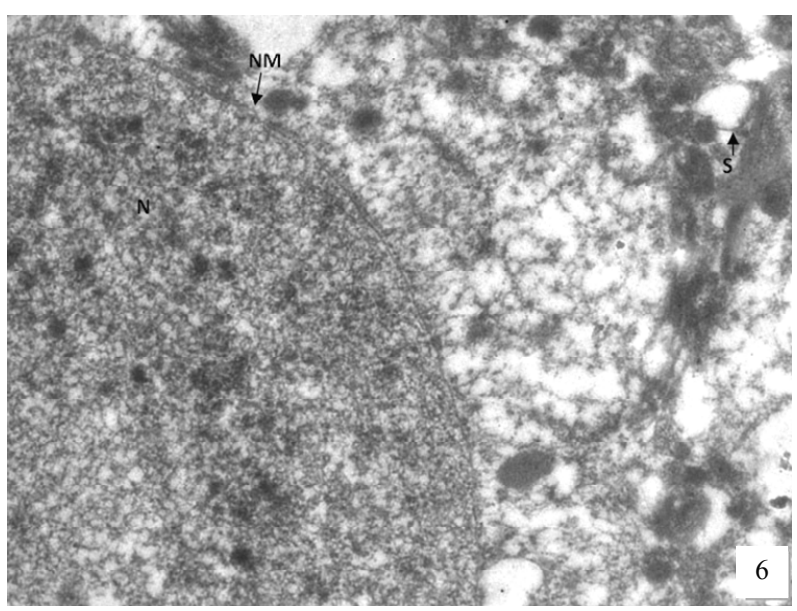

Figure 6. Electron micrograph of mercury plus melatonin rat brain nucleus revealing normal ultrastructural features (×5000).

radical production and consequently higher hydroxyl radical formation. Enhanced levels of hydroxyl radical also initiate LPO levels to rise in the brain regions. Glutathione is the most abundant low molecular weight thiol containing compound in the living cells. Its reduced form contributes to the stabilizing thiol groups of membrane enzymes and by acting as a reducing agent for hydrogen peroxide and free radicals, to protect the cells against oxidative stress [57] and detoxification of ROS [58,59]. It also participates in the enzymatic reduction of membrane hydroperoxy-phospholipids and prevents the formation of secondary alkoxyl radicals when organic peroxides are homolyzed. In the present study, reduction in GR and GPx followed by an increase in GST activity in the brain regions reflected an increased free radical (ROS) generation after mercury intoxication. Elevation in GST activity indicates the occurrence of oxidative stress status of the brain. Since GST is considered to be an oxidative stress marker, it helps in detoxification of xenobiotics by catalyzing the conjugation of electrophillic molecules with GSH [60]. Alterations in these enzymes led finally to the depletion of GSH levels resulting in oxidative damage of the nerve tissue in the present study as supported by ultrastructural studies. Ascorbic acid is a powerful reducing agent which helps in activating several enzymes and is also known as an antioxidant for detoxifying several toxic substances [61]. In mercury treated rats, the concentration of ascorbate was reduced as it helps to induce cellular GSH levels in stress condition [37]. These results thus indicate an oxidative damage caused by mercury treatment, the fact of which is proven by increased malondialdehyde formation and subsequently compensated by utilization of ascorbic acid to increase the GSH levels and to balance the LPO levels. 
As shown in our previous investigation [37], protein levels were reduced by the ingestion of mercuric chloride. Increased protein carbonyl is a marker for the oxidation of proteins. The free metal ions bind to the cations binding locations on the protein and in the presence of $\mathrm{H}_{2} \mathrm{O}_{2}$ and $\mathrm{O}_{2}$, it can transform side chain amine groups on several amino acids into carbonyls [62]. The tissue damage is further correlated by its increased accumulation of the toxicant in the brain regions indicating the development of neurodegenerative disorder caused by mercury intoxication $[63,64]$. These effects were further reconfirmed by the electron microscopic study of brain which showed discontinuous myelin sheath around the axon including swollen mitochondria, blebbed nucleus as well as nuclear membrane changes with the treatment of mercuric chloride. Mercury treatment further depletes the mitochondrial enzymes, in turn, leading to severe mitochondrial damage as documented in our earlier reports leading to cellular metabolic insult in cells and tissue $[65,66]$. These degenerative changes in the brain could again be correlated with the reduction of total proteins and lipid levels [37]. Accumulation of mercury compounds in different parts of the central nervous system (CNS) (olfactory bulbs, cerebral hemispheres, cerebellum, medulla oblongata and spinal cord) in relation to the cytoarchitectural changes in myelin sheath as well as in glycosidases levels was reported in animals. Its accumulation also resulted in degeneration and inhibition of enzymes [67] in support of our data resulting in loss of brain functions.

Numerous in vitro and in vivo studies have demonstrated the ability of melatonin to protect against free radical destructions $[29,37,51,68,69]$. Supplementation of melatonin to mercury intoxicated rats in this study was thus effective in quenching oxidative stress as well as structural changes exerted by mercury intoxication. It is well known that melatonin and its subsequent metabolites are powerful antioxidants in prevention of free radical production and quenching of these radicals by enhancing the defensive function during stress state to protect neural tissue structure and function as reported in other tissue (Rao and Bhavana, 2008; Rao et al. 2010).

\section{Conclusions}

This study reveals that inorganic mercury alters $\mathrm{CH}$ functions and structure followed by $\mathrm{C}$ and $\mathrm{MO}$ in the brain by binding to the thiol group of proteins, in turn, disturbing their various enzymatic and non enzymatic components of defense system. Subsequently, it also brought about ultrastructural changes in brain of treated rats. Co-administration of melatonin exhibited definite protective role against mercuric chloride induced brain dysfunctions due to its inherent antioxidant properties Thus, melatonin may be useful as a therapeutic agent to human population exposed to heavy metals at work places.

\section{Acknowledgements}

This work was supported by Research Grants from Gujarat State Council on Science and Technology (Gujcost), Gandhinagar, India to Prof. M. V. Rao. I would also like to acknowledge Prof. M. N. Patel for his technical advices to statistically analyze the data.

\section{REFERENCES}

[1] T. W. Clarkson, J. B. Hursh, P. R. Sager and T. L. M. Syversen, "Mercury in Biological Monitoring of Toxic Metals," Plenum Press, New York, 1988, pp. 199-246.

[2] World Health Organization (WHO), "Inorganic Mercury. In Environmental Health Criteria," Vol. 118, Geneva, 1991, pp. 1-115.

[3] J. Clausen, "Mercury and Multiple Sclerosis," Acta Neurologica Scandinavica, Vol. 87, No. 6, 1993, pp. 461-464. doi:10.1111/j.1600-0404.1993.tb04137.x

[4] M. S. Hua, C. C. Huang and Y. J. Yang, "Chronical Elemental Mercury Intoxication: Neuropsychological Follow-Up Case Study," Brain Injury, Vol. 10, No. 5, 1996, pp. 377-384. doi:10.1080/026990596124386

[5] H. Langauer-Lewowicka and M. Zajac-Nedza, "Changes in the Nervous System Due to Occupation Metallic Mercury Poisoning," Neurologia Neurochirurgia Polska, Vol. 31, No. 5, 1997, pp. 905-913.

[6] D. Deleu, V. Hanssens, H. S. Salmy and I Hastie, "Peripheral Polyneuropathy Due to Chronic Use of Topical Ammoniated Mercury," Clinical Toxicology, Vol. 36, No. 3, 1998, pp. 233-237. doi:10.3109/15563659809028945

[7] S. Gasso, C. Sunol, C. Sanfeliu, E. Rodriguez-Farre and R. M. Cristofol, "Pharmacological Characterization of the Effects of Methyl Mercury \& Mercuric Chloride on Spontaneous Nonadrenaline Release from Rat Hippocampal Slices," Life Sciences, Vol. 67, No. 10, 2000, pp. 1219- 1231. doi:10.1016/S0024-3205(00)00715-3

[8] G. Galreunthaier, W. Pialler and P. Kolanka, "Glutathione Depletion and in Vitro Lipid Peroxidation in Mercury or Malate Induced Acute Renal Failure," Biochemical Pharmacology, Vol. 32, No. 19, 1983, pp. 2969-2972. doi:10.1016/0006-2952(83)90404-5

[9] National Research Council (NRC), "Toxicological Effects of Methylmercury," National Academy Press, Washington DC, 2000, pp. 54-56.

[10] G. Discalzi, E. Pira, E. Herrero-Hernandez, C. Valentini, M. Turbiglio and F. Meliga, "Occupational Mn Parkinsonism: Magnetic Resonance Imaging and Clinical Patterns Following $\mathrm{CaNa}_{2}$-EDTA Chelation," Neurotoxicology, Vol. 21, No. 5, 2000, pp. 863-866.

[11] S. Araragi, M. Kondoh, M. Kawase, S. Saito, M. Higashimoto and M. Sato, "Mercuric Chloride Induces Apoptosis via a Mitochondrial-Dependent Pathway in Human Leukemia Cells," Toxicology, Vol. 184, No. 1, 2003, pp. 


\section{1-9. doi:10.1016/S0300-483X(02)00443-2}

[12] F. L. Lorscheider, M. J. Vimy, A. O. Summers and H. Zwiers, "Inorganic Mercury and the CNS: Genetic Linkage of Mercury and Antibiotic Resistance," Toxicology, Vol. 97, No. 1-3, 1995, pp. 19-22. doi:10.1016/0300-483X(94)02964-V

[13] J. C. Pendergrass and B. E. Haley, "The Toxic Effects of Mercury on CNS Proteins: Similarity to Observations in Alzheimer's Disease," University of Kentucky, Lexington, 1997, IAOMT Symposium Paper.

[14] C. Hock, G. Drasch, S. Golombowski, F. Muller-Spahn, B. Willer-Shausen-Zonnchen, P. Schwarz, U. Hock, J. H. Growdon and R. M. Nitsch, "Increased Blood Mercury Levels in Patients with Alzheimer's Disease," Journal of Neural Transmission, Vol. 105, No. 1, 1998, pp. 59-68. doi: $10.1007 / \mathrm{s} 007020050038$

[15] J. C. Pendergrass and B. E. Haley, "Mercury Vapor Inhalation Inhibits Binding of GTP-Similarity to Lesions in Alzheimer's Diseased Brains," Neurotoxicology, Vol. 18, No. 2, 1997, pp. 315-24.

[16] H. Moreno-Fuenmayor, L. Borjas, A. Arrieta and V. Valera, "Plasma Excitatory Amino Acids in Autism," Investigación Clínica, Vol. 37, No. 2, 1996, pp. 113-128.

[17] S. Belletti and R. Gatti, "Time Course Assessment of Methylmercury Effects on C6 Glioma Cells: Submicromolar Concentrations Induce Oxidative DNA Damage and Apoptosis," Journal of Neuroscience Research, Vol. 70, No. 5, 2002, pp. 703-711. doi:10.1002/jnr.10419

[18] F. Bakir, S. F. Damluji, L. Amin-Zaki, M. Murtadha, A. Khalidi and N. Y. Al-Rawi, "Methyl Mercury Poisoning in Iraq," Science, Vol. 181, No. 4096, 1973, pp. 230-241. doi:10.1126/science.181.4096.230

[19] T. Kjelstrom, P. Kennedy, S. Wallis, A. Stewart, L. Friberg and B. Lind, "Physical and Mental Development of Children with Prenatal Exposure to Mercury from Fish. Stage II: Interviews and Psychological Tests at Age 6," National Swedish Environmental Protection Board Report, Solna, 1989.

[20] P. Grandjean, P. Weihe, R. F. White, F. Debes, S. Araki and K. Yokoyama, "Cognitive Deficit in 7-Year-Old Children with Prenatal Exposure to Methyl Mercury," Neurotoxicology and Teratology, Vol. 19, No. 6, 1997, pp. 417428. doi:10.1016/S0892-0362(97)00097-4

[21] P. Grandjean, E. Budtz-Jorgensen, R. F. White, P. J. Jorgensen, P. Weihe and F. Debes, "Methyl Mercury Exposure Biomarkers as Indicators of Neurotoxicity in children Aged 7 Years," American Journal of Epidemiology, Vol. 150, No. 3, 1999, pp. 301-305.

[22] G. L. Diamond and R. K. Zalups, "Understanding Renal Toxicity of Heavy Metals," Toxicologic Pathology, Vol. 26, No. 1, 1998, pp. 92-103. doi:10.1177/019262339802600111

[23] H. Fukine, M. Hirai, Y. M. Rsuch and Y. Yamane, "Effect of Zinc Pretreatment on Mercuric Chloride-Induced Lipid Peroxidation in the Rat Kidney," Toxicology and Applied Pharmacology, Vol. 73, No. 3, 1984, pp. 395-401. doi:10.1016/0041-008X(84)90091-7
[24] N. Basu, A. M. Scheuhammer, R. D. Evans, M. O’Brien and L. H. M. Chan, "Cholinesterase and Monoamine Oxidase Activity in Relation to Mercury Levels in the cerebral Cortex of Wild River Otters," Human and Experimental Toxicology, Vol. 26, No. 3, 2007, pp. 213-220. doi:10.1177/0960327107070570

[25] N, Basu, A. M. Scheuhammer, K. Rouvinen-Watt, R. D. Evans, N. Grochowina and L. H. Chan, "The Effects of Mercury on Muscarinic Cholinergic Receptor Subtypes (M1 and M2) in Captive Mink," Neurotoxicology, Vol. 29, No. 2, 2008, pp. 328-334. doi:10.1016/j.neuro.2008.01.003

[26] D. X. Tan, L. D. Chen, B. Poeggeler, L. C. Manchester and R. J. Reiter, "Melatonin: A Potent, Endogenous Hydroxyl Radical Scavenger," Journal of Endocrinology, Vol. 1, 1993, pp. 57-60.

[27] M. Allegra, R. J. Reiter, D. X. Tan, C. Gentile, L. Tesoriere and M. A. Livrea, "The Chemistry of Melatonin Interaction with Reactive Species," Journal of Pineal Research, Vol. 34, No. 1, 2003, pp. 1-10. doi:10.1034/j.1600-079X.2003.02112.x

[28] R. J. Reiter, D. X. Tan, J. C. Mayo, R. M. Sainz, J. Leon, Z. Czarnocki, "Melatonin as an Antioxidant; Biochemical Mechanisms and Pathophysiological Implications," Acta Biochimica Polonica, Vol. 50, No. 4, 2003, pp. 1129-1146.

[29] I. Antolin, C. Rodriguez, R. M. Sainz, J. Mayo, H. Uria, M. L. Kotler, M. J. Rodriguez-Colunga, D. Tolivia and A. Menendez Pelaez, "Neurohormone Melatonin Prevents Cell Damage: Effect on Gene Expression for Antioxidant Enzymes," The FASEB Journal, Vol. 10, No. 8, 1996, pp. 882-890.

[30] C. Rodriguez, J. C. Mayo, R. M. Sainz, I. Antolin, F. Herrera, V. Martin and R. J. Reiter, "Regulation of Antioxidant Enzymes: A Significant Role for Melatonin," Journal of Pineal Research, Vol. 36, No. 1, 2004, pp. 1-9. doi:10.1046/j.1600-079X.2003.00092.x

[31] C. Tomas-Zapico and A. Coto-Montes, "A Proposed Mechanism to Explain the Stimulatory Effect of Melatonin on Antioxidative Enzymes," Journal of Pineal Research, Vol. 39, No. 2, 2005, pp. 99-104. doi:10.1111/j.1600-079X.2005.00248.x

[32] K. Winiarska, T. Fraczyk, D. Malinska, J. Drozak and J. Bryla, "Melatonin Mitigated Diabetes Induced Oxidative Stress in Rabbits," Journal of Pineal Research, Vol. 40, No. 2, 2006, pp. 168-176. doi:10.1111/j.1600-079X.2005.00295.x

[33] J. Leon, D. Acuna-Castroviejo, G. Escames, D. X. Tan and R. J. Reiter, "Melatonin Mitigates Mitochondrial Malfunction," Journal of Pineal Research, Vol. 38, No. 1, 2005, pp. 1-9. doi:10.1111/j.1600-079X.2004.00181.x

[34] S. Lopez-Burillo, D. X. Tan, J. C. Mayo, R. M. Sainz and R. J. Reiter, "Melatonin Xanthurenic Acid Reveratrol, EGCG, Vitamin C and Alpha-Lipoic Acid Differentially Reduce Oxidative DNA Damage Induced by Fenton Reagents, a Study of Their Individual and Synergistic Actions," Journal of Pineal Research, Vol. 34, No. 4, 2003, pp. 269-277. doi:10.1034/j.1600-079X.2003.00041.x

[35] R. J. Reiter, D. Melchiorri, E. Sewerynek, B. Poeggeler, 
L. Barlow-Walden, J. Chuang, G. G. Ortiz and D. A. Acuna-Castroviejo, "A Review of the Evidence Supporting Melatonin'S Role as an Antioxidant," Journal of Pineal Research, Vol. 18, No. 1, 1995, pp. 1-18. doi:10.1111/j.1600-079X.1995.tb00133.X

[36] M. V. Rao, A. R. Purohit and T. A. Patel, "Melatonin Protection on Mercury Exerted Neurotoxicity in the Rat," Drug and Chemical Toxicology, Vol. 33, No. 2, 2010, pp. 209-216. doi:10.3109/01480540903349258

[37] G. Jansson and Harms-Ringdahl, "Stimulating Effects in Human Polymorphonuclear Leucocytes," Free Radical Research, Vol. 18, No. 2, 1993, pp. 87-98. doi:10.3109/10715769309147345

[38] B. O. Lund, D. M. Miller and J. S. Woods, "Studies on $\mathrm{Hg}$ (II)-Induced $\mathrm{H}_{2} \mathrm{O}_{2}$ Formation and Oxidative Stress in Vivo and Vitro in Rat Kidney Mitochondria," Biochemical Pharmacology, Vol. 45, No. 10, 1993, pp. 2017-2024. doi:10.1016/0006-2952(93)90012-L

[39] R. J. Lewis and R. Tatken, "NIOSH. Registry of Toxic Effects of Chemical Substances," US Department of Health, Education and Welfare, Cincinati, 1979.

[40] O. Vakkuri, J. Leppaluto and A. Kaupplia, "Oral Administration and Distribution of Melatonin in Human Saliva and Urine," Life Sciences, Vol. 37, No. 5, 1985, pp. 489495. doi:10.1016/0024-3205(85)90412-6

[41] H. Ohkawa, N. Ohishi and K. Yagi, "Assay for Lipid Peroxides in Animal Tissue by Thiobarbituric Acid Reaction," Analytical Biochemistry, Vol. 95, No. 2, 1979, pp. 351-358. doi:10.1016/0003-2697(79)90738-3

[42] G. L. Ellman, "Tissue Sulfhydyl Groups," Archives of Biochemistry and Biophysics, Vol. 82, No. 1, 1959, pp. 70-77. doi:10.1016/0003-9861(59)90090-6

[43] J. T. Rotruck, A. L. Pope, H. E. Ganther, A. B. Swanson, D. G. Hafeman and W. G. Hoekstra, "Selenium: Biochemical Roles as a Component of Glutathione Peroxidase," Science, Vol. 179, No. 4073, 1973, pp. 585-590. doi:10.1126/science.179.4073.588

[44] I. Carlberg and B. Mannervik, "Glutathione Reductase," Methods in Enzymology, Vol. 113, 1985, pp. 484-490. doi:10.1016/S0076-6879(85)13062-4

[45] W. H. Habig, J. P. Michael and W. B. Jakoby, "Glutathione S-Transferases. The First Enzymatic Step in Mercapturic Acid Formation," The Journal of Biological Chemistry, Vol. 249, No. 22, 1974, pp. 7130-7139.

[46] R. L. Levine, J. A. Williams, E. R. Stadtman and E. Shacter, "Carbonyl for Determination of Oxidatively Modified Proteins," Methods in Enzymology, Vol. 233, 1994, pp. 346-357. doi:10.1016/S0076-6879(94)33040-9

[47] E. Pick and Y. Keisari, "Superoxide Anion and Hydrogen Peroxide Production by Chemically Elicited Peritoneal Macrophages-Induction by Multiple Non Phagocytic Stimuli," Cellular Immunology, Vol. 59, No. 2, 1981, pp. 301-318. doi:10.1016/0008-8749(81)90411-1

[48] O. H. Lowry, N. J. Rosebrough, A. L. Farr and R. J. Randall, "Protein Measurements with Folin Phenol Reagent," The Journal of Biological Chemistry, Vol. 193, 1951, pp. 265-275.
[49] J. H. Roe and C. A. Kuether, "The Determination of Ascorbic Acid in Whole Blood and Urine through the 2,4-Dinitrophenyl Hydrazine Derivatives of Dehydroascorbic Acid," The Journal of Biological Chemistry, Vol. 147, 1943, pp. 399-407.

[50] M. V. Rao and H. Tiwari, "Amelioration of Melatonin of Chromosomal Anomalies Induced by Arsenic and/or Fluoride in Human Blood Lymphocyte Culture," Fluoride, Vol. 39, No. 4, 2006, pp. 251-256.

[51] G. Lumb, "Metal Toxicity," In: J. E. Craighead, Ed., Pathology of Environmental and Occupational Disease, Mosby Year Book, St. Louis, 1995.

[52] H. L. Evans, "Environmental and Occupational Medicine," Lippincott-Raven, Philadelphia, 1998.

[53] B. Korenekova, M. Skalickaand and P. Nad, "Cadmium Exposure of Cattle After Long-Term Emission from Polluted Area," Trace Elements and Electrolytes, Vol. 19, No. 2, 2002, pp. 97-99.

[54] M. V. Rao, "Mercury and Its Effect on Mammalian Systems. A Critical Review," Indian Journal of Environment and Toxicology, Vol. 7, No. 1, 1997, pp. 3-11.

[55] Y. L. Huang, S. L. Cheng and T. H. Lin, "Lipid Peroxidation in Rats Administrated with Mercuric Chloride," Biological Trace Element Research, Vol. 52, No. 2, 1996, pp. 193-206. doi:10.1007/BF02789461

[56] E. Hijova, F. Nistiar and A. Sipulova, "Changes in Ascorbic Acid and Malondialdehyde in Rats after Exposure to Mercury," Bratislavské Lekárske Listy, Vol. 106, No. 8-9, 2005, pp. 248-251.

[57] F. H. El-Rashidy, W. A. Al-Turk and S. J. Stohs, "Glutathione, Glutathione Reductase and Glutathione S-Transferase Activities in Erythrocytes and Lymphocytes in Chronic Renal Disease," Research Communications in Chemical Pathology and Pharmacology, Vol. 44, No. 3, 1984, pp. 423-429.

[58] D. J. Reed, "Glutathione: Toxicological Implications," Annual Review of Pharmacology and Toxicology, Vol. 30, 1990, pp. 603-631. doi:10.1146/annurev.pa.30.040190.003131

[59] R. Franco, O. J. Schoneveld, A. Pappa and M. I. Panayiotidis, "The Central Role of Glutathione in the Pathophysiology of Human Diseases," Archives of Physiology and Biochemistry, Vol. 113, No. 4-5, 2007, pp. 234-258. doi:10.1080/13813450701661198

[60] V. H. Neefjes, C. T. Evelo, L. G. Baars and L. E. Blanco, "Erythrocyte Glutathione S Transferase as a Marker of Oxidative Stress at Birth," Archives of Disease in Childhood: Fetal and Neonatal Edition, Vol. 81, No. 2, 1999, pp. 130-133. doi:10.1136/fn.81.2.F130

[61] R. J. Kutsky, "Handbook of Vitamins and Hormones," Van Nostrand Reinhold, New York, 1973.

[62] B. Halliwell, "Free Radicals, Proteins and DNA: Oxidative Damage versus Redox Regulation," Biochemical Society Transactions, Vol. 24, No. 4, 1996, pp. 1023-1027.

[63] S. Hussain, D. A. Rodgers, H. M. Duhart and S. F. Ali, "Mercuric Chloride-Induced Reactive Oxygen Species 
and Its Effect on Antioxidant Enzymes in Different Regions of Rat Brain," Journal of Environmental Science and Health, Vol. 32, No. 3, 1997, pp. 359-409.

[64] T. W. Clarkson, "The Three Modern Faces of Mercury," Environmental Health Perspectives, Vol. 110, No. 1, 2002, pp. 11-23. doi:10.1289/ehp.02110s111

[65] M. V. Rao and B. Gangadharan, "Antioxidative Potential of Melatonin against Mercury Induced Intoxication in Spermatozoa in Vitro," Toxicology in Vitro, Vol. 22, No. 4, 2008, pp. 935-942. doi:10.1016/j.tiv.2008.01.014

[66] M. V. Rao and B. Chhunchha, "Effects of Melatonin on Mercury Induced Endocrine Toxicity in the Rats," Journal of Herbal Medicine and Toxicology, Vol. 3, No. 2, 2009, pp. 85-89.

[67] S. D. Vinay, K. G. Raghu and P. P. Sood, "Dose and Dura-

\section{Abbreviations}

MLT: Melatonin

HD: High Dose

LD: Low Dose

Mi: Mitochondria

My: Myelinated Nerve Fibre tion Related Methyl Mercury Deposition, Glucosidase Inhibition, Myelin Degeneration and Chelating Therapy," Molecular and Cellular Biology, Vol. 36, No. 5, 1990, pp. 609-623.

[68] R. J. Reiter, D. X. Tan, L. C. Manchester and I. L. Tamura, "Melatonin Defeats Neutrally-Derived Free Radicals and Reduces the Associated Neuromorphological and Neurobehavioral Damage," Journal of Physiology and Pharmacology, Vol. 38, 2007, pp. 3-22.

[69] Y. Quiroz, A. Ferrebuz, F. Romero, N. D. Vaziri and R. I. Bernardo, "Melatonin Ameliorates Oxidative Stress, InFlammation, Proteinuria, and Progression of Renal Damage in Rats with Renal Mass Reduction," American Journal of Physiology: Renal Physiology, Vol. 294, No. 2, 2008, pp. 336-344. doi:10.1152/ajprenal.00500.2007

NMy: Non Myelinated Nerve Fibre

$\mathrm{N}$ : Nucleus

NM: Nuclear Membrane

S: Intracellular Space 